\title{
Performance des services publics municipaux : entre outils du contrôle de gestion et facteurs contingents
}

\author{
Donatien Avelé, D. sc. De gestion \\ Université de Moncton
}

\section{INTRODUCTION}

\section{Le problème de l'organisation est d'obtenir la coopération entre un grand nombre d'individus ou d'unités qui partagent seulement et partiellement des objectifs convergents.}

« Le problème de l'organisation est d'obtenir la coopération entre un grand nombre d'individus ou d'unités qui partagent seulement et partiellement des objectifs convergents $»$. Cette remarque est particulièrement marquée par des frontières internes. En particulier, l'opposition qui sépare les administrations et les techniciens est ancienne et profonde. Elle se manifeste, soit par une ignorance polie, soit par des dissensions entre des hommes ayant des formations et des logiques différentes, voire contradictoires ${ }^{2}$. Auparavant, le pouvoir se trouvait essentiellement entre les mains des techniciens, dont dépendaient les grandes réalisations si chères aux élus. Aujourd'hui, le secrétaire général, le directeur financier et le contrôleur de gestion semblent occuper une place de plus en plus importante $^{3}$. Ils ont la responsabilité de la maîtrise des charges, la réduction des coûts afin de dégager un maximum d'autofinancement pour réaliser ces gros investissements, toujours aussi chers aux élus ${ }^{4}$. L'écart grandissant qui sépare les techniciens des administratifs produit un choc de cultures. Les principes de gestion, l'adoption d'une approche mercatique et d'un

\section{APPORTS THÉORIQUES}

Une des caractéristiques des organisations publiques et en particulier des collectivités locales est leur complexité. Celle-ci est, entre autres, le fruit de l'hétérogénéité des activités. Une commune possède un grand nombre de système de contrôle de gestion incitent les élus à être plus à l'écoute des spécialistes et des gestionnaires ${ }^{5}$. Les élus font ensuite exécuter leurs choix, établis à partir des avis du secrétaire général, qui devient le véritable directeur général de l'organisation municipale, du directeur des services financiers, qui détermine la possibilité de financer le projet, et du directeur des services techniques, qui n'est plus le seul à apporter des informations utiles à la prise de décision ${ }^{6}$.

Cet article a pour objet de fournir une description de l'état des lieux des pratiques des municipalités existantes afin de contribuer à la connaissance des outils de contrôle de gestion nécessaire au suivi des performances dans ces entités locales. L'étude est menée à partir des données collectées par questionnaire auprès des 60 services visités constituant l'échantillon. Les apports théoriques et la méthodologie adoptée sont d'abord présentés; les résultats empiriques sont ensuite analysés et discutés.

\section{Les principes de gestion, l'adoption d'une approche mercatique et d'un système de contrôle de gestion incitent les élus à être plus à l'écoute des spécialistes et des gestionnaires.}

services nécessaires au bon fonctionnement de la collectivité mais répondant à des besoins extrêmement variés n'ayant aucun lien apparent entre eux. Un des rôles du contrôle de gestion et tout spécialement la comptabilité analytique va 
être de tenter à mieux coordonner l'ensemble de ces activités diverses. Mais cette hétérogénéité conduit également à s'interroger sur la pertinence d'un système unique et unificateur. Le contrôle de gestion présente de fait une double facette : une dimension technico-économique de nature comptable et financière complétée d'une dimension "managériale, psychologique » liée à la notion de contrôle et d'influence des comportements ${ }^{7}$.

Toutefois, la pratique du contrôle de gestion met effectivement en évidence l'importance de l'animation des hommes. La fonction requiert l'implication de tous et un certain degré de motivation. Elle est également censée développer l'autonomie des responsables ${ }^{8}$. Ces éléments sont particulièrement présents au sein d'un des outils phares du contrôle de gestion à savoir le tableau de bord. En tant que dispositif souple et diversifié, le tableau de bord peut se définir comme une présentation, à partir d'indicateurs sélectionnés d'un suivi de facteurs clefs de gestion de l'entité concernée?

S'agissant de la responsabilisation des agents communaux, certains auteurs font référence à la notion de responsabilité transposée au monde de l'entreprise. Cette notion présente ainsi deux facettes distinctes, correspondant à deux modes gestionnaires opposés : la décentralisation et son contraire $^{10}$.

\section{Les marges de manœuvre sont réelles et la possibilité d'isoler la responsabilité du manager existe. En revanche, dans une dynamique de centralisation fortement présente en milieu public, la notion de responsabilité a un champ d'action plus hiérarchique et précise. L’autorité centrale impose une obligation de résultat.}

Dans le premier cas, la responsabilité recouvre une définition large. Elle renvoie l'idée de maîtrise d'un domaine délimité. Les marges de manœuvre sont réelles et la possibilité d'isoler la responsabilité du manager existe. En revanche, dans une dynamique de centralisation fortement présente en milieu public, la notion de responsabilité a un champ d'action plus hiérar- chique et précis. L'autorité centrale impose une obligation de résultat (fixation d'objectifs et évaluation de performances). Pour l'équipe dirigeante, le tableau de bord est utilisé comme " moyen de légitimation " ${ }^{11}$, des politiques imposées. En effet, son implantation repose sur un discours de modernisation, de rationalisation au nom duquel les activités doivent être conduites. L'introduction de cet outil souligne les objectifs généraux de la mairie et recherche l'adhésion de ses membres. Plusieurs agents parlent spontanément de changement de culture de gestion " affiché dès le départ ». "C'est une volonté politique en terme de management ».

\section{Le tableau de bord est par nature un outil personnalisé dans son contenu puisque étant construit sur les variables clefs d'action, particulières à chaque manager en management suppose déjà de parvenir à préciser les objectifs de manière suffisante.}

L'utilisation du tableau de bord en vue de construire un outil sur-mesure en adéquation aux objectifs de performances devient une pratique courante dans le secteur public. Ainsi, le tableau de bord est par nature un outil personnalisé dans son contenu puisque étant construit sur les variables clefs d'action, particulières à chaque manager en management suppose déjà de parvenir à préciser les objectifs de manière suffisante. "Mais la pluralité de missions de service public, notamment dans une mairie, limite l'exercice. Les objectifs ont alors tendance à rester dans le flou ${ }^{12}$.

En ce qui concerne l'évaluation de la performance à travers l'usage des tableaux de bord dans les mairies, le contrôle de gestion s'inscrit explicitement dans cette démarche responsabilisante. Dans la littérature, le croisement entre contrôle de gestion et responsabilisation, fait référence d'une part, à l'appropriation et à la maîtrise de la performance et, d'autre part, au système d'animation permettant de fixer et d'atteindre les objectifs ${ }^{13}$. La mesure de la performance peut se réaliser à l'aide du contrôle d'animation stipulant la responsabilisation. Selon cette optique, l'encadrement joue un rôle déterminant $^{14}$. La responsabilisation constitue 
même une étape à part entière de l'établissement du contrôle de gestion (après celle de fixation des objectifs et avant celle de management opérationnel $)^{15}$. Il s'agit de décliner des objectifs afin de permettre à l'ensemble des managers locaux de gérer la performance. La responsabi-

\section{MÉTHODOLOGIE DE L’ÉTUDE}

Le contexte des pays de l'Afrique subsaharienne influence considérablement les choix méthodologiques des recherches en gestion. De nombreux problèmes particuliers se posent à tout chercheur dans ce domaine et le contraignent à adopter des stratégies de recherche appropriées afin de pouvoir disposer de données primaires nécessaires à sa recherche. Parmi ces problèmes on peut citer les difficultés d'accès à l'information, les obstacles de l'environnement, la faible fiabilité des données statistiques et le manque de collaboration des populations cibles. Ensuite, on pourrait ajouter les difficultés découlant de l'impératif d'une recherche confrontée au terrain ${ }^{18}$. L'enquête a été menée dans lisation les concerne afin de rendre les décisions cohérentes aux objectifs stratégiques ${ }^{16}$. Nous retrouvons là une des missions classiques du contrôle organisationnel et, pour ce faire, les responsables locaux doivent connaître les objectifs et être incités à les respecter ${ }^{17}$.

les services publics municipaux des villes camerounaises. Ainsi, pour mieux appréhender l'objet de l'étude, 148 questionnaires ont été administrés aux responsables de différents services publics municipaux des villes camerounaises. Sur les 148 questionnaires expédiés, 88 sont retournés, parmi lesquels 28 sont non exploitables et 60 sont exploitables, tel que précisé au tableau 1. Enfin, une identification des outils de gestion utilisés dans les municipalités visitées et nécessaires au suivi de la performance communale a été réalisée. Toutes les données ont été analysées avec le logiciel SPSS version 10.00 .

Tableau 1 : Taux de réponse global de l'enquête quantitative

\begin{tabular}{|l|c|c|}
\hline & Totaux & Pourcentage \\
\hline Questionnaires envoyés & 148 & $100 \%$ \\
\hline Questionnaires retournés & 88 & $59,45 \%$ \\
\hline Questionnaires non exploitables & 28 & $18,91 \%$ \\
\hline Questionnaires exploitables & 60 & $40,54 \%$ \\
\hline
\end{tabular}




\section{PRINCIPAUX RÉSULTATS OBTENUS ET DISCUSSION}

L'analyse de la performance municipale ou encore le contrôle des activités au sein des mairies camerounaises peut très souvent s'exercer de façon directe ou informelle sans véritablement nécessiter d'outils de gestion sophistiqués à l'usage que l'on peut tirer des informations recueillies dans le cadre de l'enquête de terrain.

Les statistiques descriptives réalisées à partir des données recueillies lors de l'enquête quantitative révèlent que les outils de contrôle de la performance des services municipaux des villes camerounaises sont peu significatifs et restent très sommaires dans l'ensemble des 60 services visités constituant notre échantillon. En dehors de la comptabilité d'engagement qui reste généralisée dans l'ensemble des municipalités au regard des résultats de la première phase d'observation, les résultats de l'enquête quantitative sont très peu significatifs par rapport aux autres outils de gestion utilisés dans ces mêmes municipalités. L'analyse de la performance municipale ou encore le contrôle des activités au sein des mairies camerounaises peut très souvent s'exercer de façon directe ou informelle sans véritablement nécessiter d'outils de gestion sophistiqués à l'usage que l'on peut tirer des informations recueillies dans le cadre de l'enquête de terrain. L'absence des tableaux de bord dans les communes rurales peut sembler logique. Par contre le fait que la majorité des mairies relevant des grandes villes de Douala et de Yaoundé ne procède pas à une saisie des informations de gestion sous forme de tableau de bord, peut sembler tout inquiétant quant à la notion même de la performance communale. Le graphique 1 illustre l'existence des outils de gestion au sein des mairies camerounaises.

\section{Graphique 1 : Existence d'outils de gestion dans les 60 services municipaux}

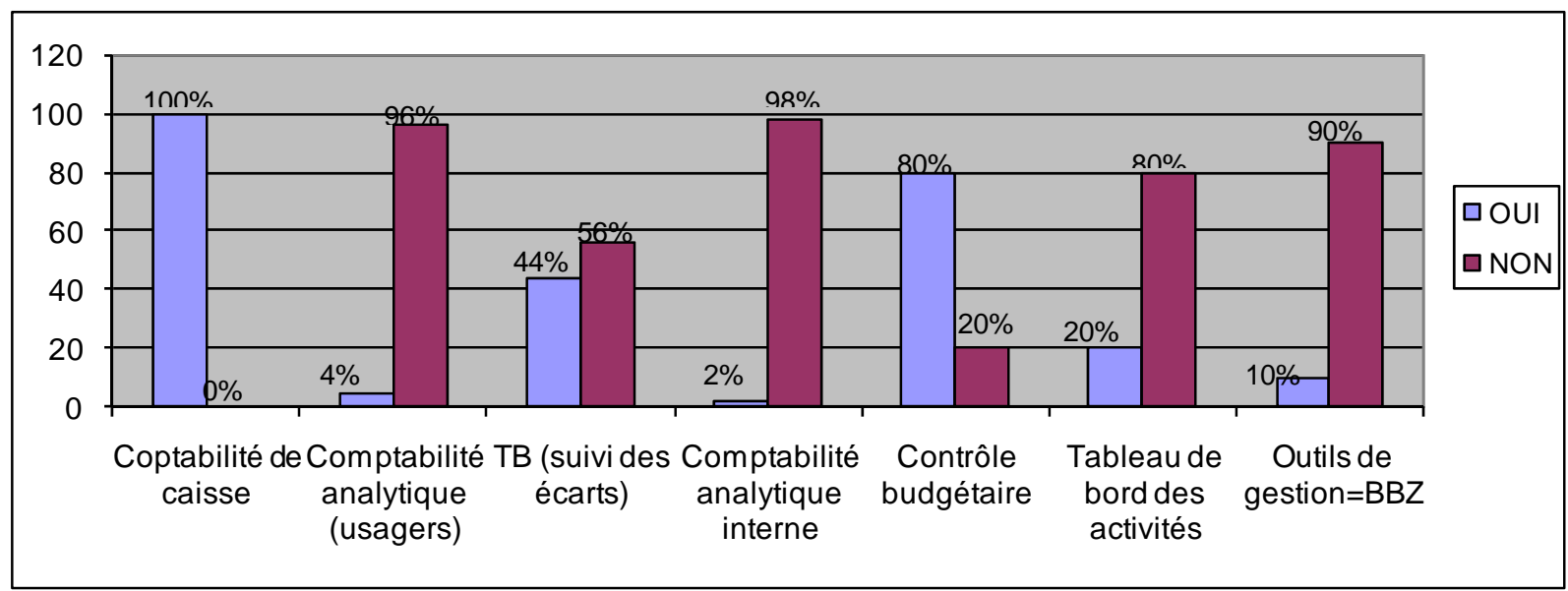

En définitive, nous avons observé, comme le tableau le montre si bien, que la comptabilité de caisse est très répandue dans tous les 60 services de l'échantillon. Dans la pratique, le Budget Base Zéro (BBZ) est une méthode de mobilisation des services souple et adaptable. Il permet de faire avancer l'ensemble des services en même temps ou travailler par groupes. Enfin, en fonction de la situation financière de la ville, il est possible de mettre l'accent sur la maîtrise des coûts ou l'amélioration de la qualité des prestations. L'intérêt de la méthode réside dans la rigueur et dans la formalisation des différentes étapes qui permettent au secrétaire général de bien maîtriser l'action et dans le fait qu'elle prend appui sur les chefs de service. Le BBZ devient un langage commun de la mairie. Ainsi, les résultats de l'enquête montrent que $90 \%$ des 
mairies enquêtées n'ont pas recours au BBZ comme méthode d'élaboration des budgets communaux contre $10 \%$ qui l'utilisent pour élaborer leurs budgets.

Les résultats de l'enquête montrent que la fréquence d'élaboration des tableaux de bord dans les 60 services de l'échantillon la plus répandue est annuelle (58,3\%). Il y a une forte variation d'élaboration des tableaux de bord, même si l'on observe que $3,4 \%$ des services optent pour une élaboration trimestrielle et mensuelle des tableaux de bord $(1,7 \%$ trimestrielle et $1,7 \%$ mensuelle). Le graphique 2 fournit plus de détails quant à l'élaboration des tableaux de bord au sein des services municipaux dont la périodicité varie de " annuelle à quotidienne ».

\section{Graphique 2 : Fréquence d'élaboration des tableaux de bord dans les 60 services municipaux}

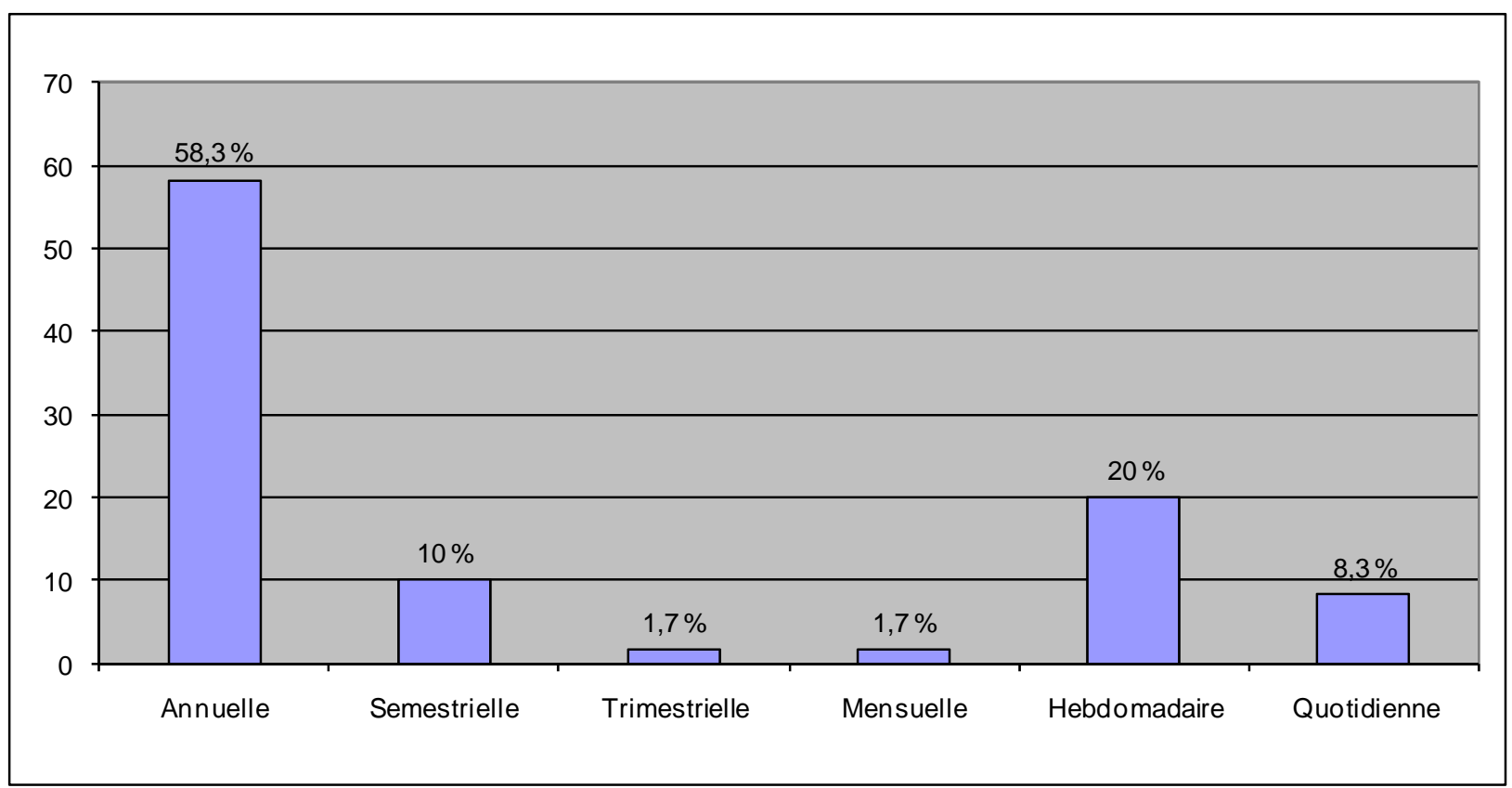

Moyenne : 10,00 maximums : 35; Écart-type : 12,89 médianes : 5,50

L'exécution du budget concentre l'essentiel de la comptabilité administrative de l'ordonnateur. C'est l'ensemble des actes posés et opérations conduites pendant la période d'exécution du budget communal qui constitue la comptabilité de l'ordonnateur. Elle permet à ce dernier de liquider et d'ordonnancer les dépenses engagées au cours de l'exercice et au receveur municipal de vérifier, prendre en compte, recouvrer ou régler les titres (perceptions ou paiement). Ainsi, les résultats de l'enquête montrent que le suivi des budgets communaux des villes camerounaises se fait beaucoup plus trimestriellement (96\%) et mensuellement (74\%). De l'autre côté, on observe que $18 \%$ des mairies suivent leurs budgets annuellement contre $4 \%$ qui le suivent semestriellement. En ce qui concerne le suivi quotidien, $30 \%$ des mairies optent pour ce procédé contre $20 \%$ pour un suivi hebdomadaire. Le graphique 3 illustre bien les détails des suivis budgétaires dans l'ensemble des mairies de l'échantillon.

\section{L'exécution du budget concentre l'essentiel de la comptabilité administrative de \\ l'ordonnateur. C'est l'ensemble des actes posés et opérations conduites pendant la période d'exécution du budget communal qui constitue la comptabilité de l'ordonnateur.}




\section{Graphique 3 : Le suivi budgétaire des mairies de l'échantillon}

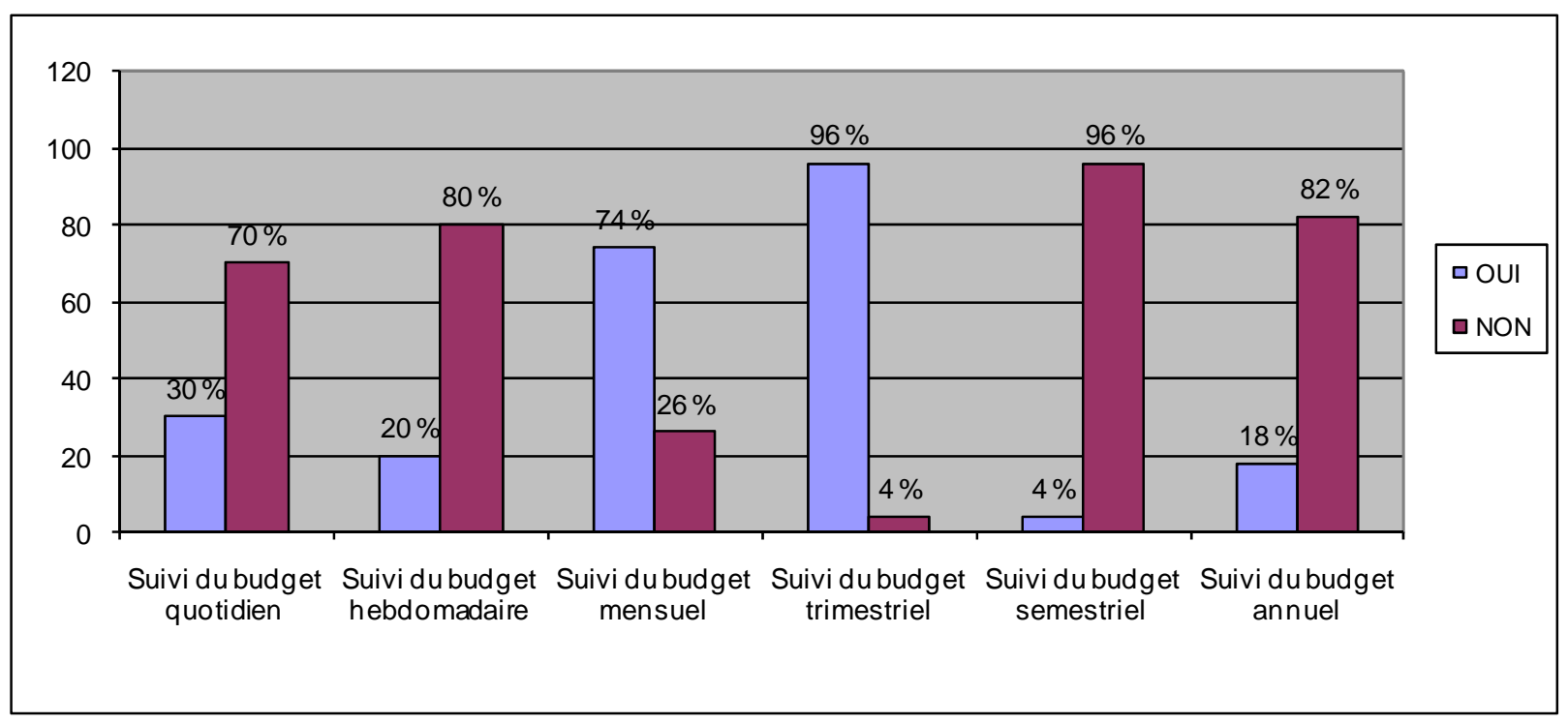

\section{CARACTÉRISTIQUES CONTINGENTES}

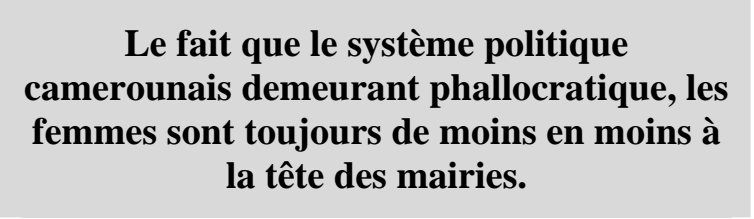

Nous présentons les caractéristiques de contingence d'ordre comportemental du chef de service liées au sexe, à l'âge, au niveau de formation et à l'ancienneté. Comme le montre le graphique 4, parmi les 60 responsables des services publics communaux visités, 20 sont de sexe masculin $(33,33 \%)$ et 40 sont de sexe féminin $(66,7 \%)$. Contrairement à la répartition des maires enquêtés de l'échantillon selon leur sexe où l'on a noté $4 \%$ des femmes, celle des 60 services publics municipaux visités est représentée par 40 femmes; ce qui est véritablement significatif. Ceci peut s'expliquer tout simplement par le fait que le système politique Camerounais demeurant phallocratique, les femmes sont toujours de moins en moins à la tête des mairies. Tel n'est pas le cas des différents responsables de services qui ont été enquêtés, car ces derniers ne sont pas des élus du peuple et ne sauront être sanctionnés par la voix du vote. Seuls la compétence, le niveau et le type de formation peuvent les conduire à des niveaux de responsabilité aussi importants.

\section{Graphique 4 : Distribution des chefs de service selon leur sexe}

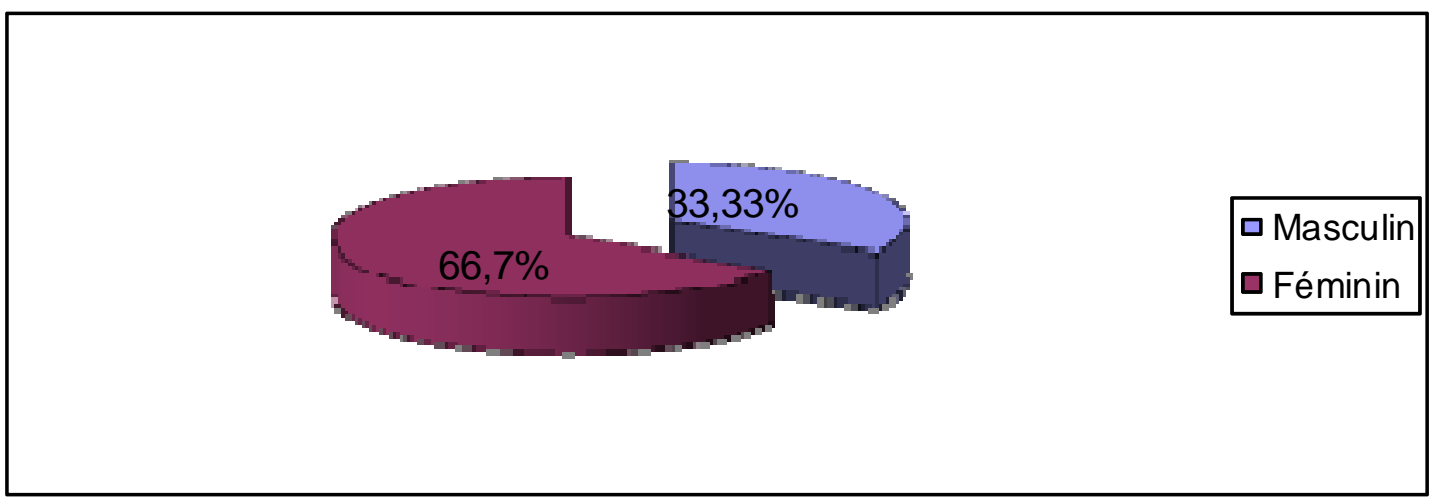


Les résultats de la première enquête montraient déjà que les chefs de services les plus jeunes parmi les 60 enquêtés avaient moins de 30 ans. Ceci vient ainsi confirmer les résultats de la deuxième enquête car, $3,3 \%$ des chefs de services ont effectivement moins de 30 ans. La plupart des chefs de services ont entre 40 et 50 ans $(56,7 \%)$. En revanche, aucun chef de service parmi les services ayant accepté de nous accueillir n'atteint l'âge de 60 ans. Ceci peut s'expliquer par le fait que les agents communaux prennent généralement leur retraite au Cameroun entre 50 et 55 ans. D'où un pourcentage de $6,7 \%$ (Graphique 5) représentant des responsables de services ayant entre 50 et 60 ans donc prêts à la retraite.

\section{Graphique 5 : Distribution des chefs de services selon leur âge}

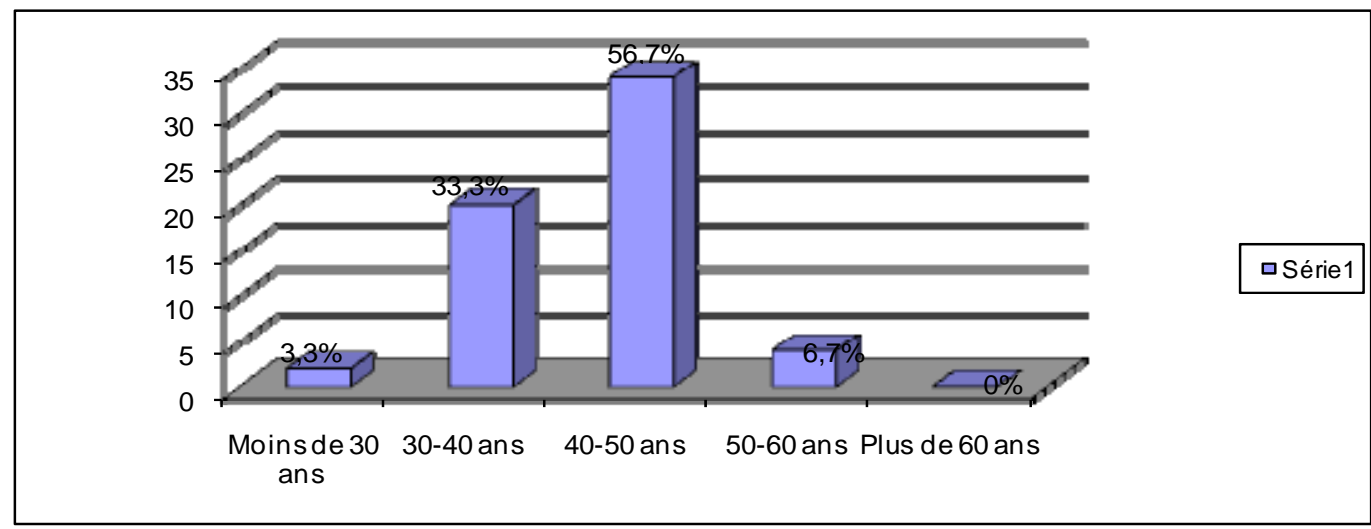

Le graphique 6 nous offre une véritable distribution des chefs de services selon leur niveau de formation parmi les mairies visitées. Ainsi, 23,3\% des responsables des services visités ont un niveau de formation $\mathrm{Bac}+3$. Ils sont dans la plupart des cas des responsables de services financiers ou de l'administration générale. Parmi les 14 responsables de services ayant un niveau de formation égal $\mathrm{Bac}+3$, on retrouve généralement des receveurs municipaux, des comptables matières tous formés au CEFAM (centre de formation de l'administration municipale) au Cameroun. Ces responsables formés par le CEFAM relèvent du ministère des finances et sont conjointement nommés par le ministère de l'administration territoriale et de la décentralisation (MINATD) et le ministère de l'économie et des finances (minefi).Toutefois, on retrouve quelques cadres financiers mutés par le minefi et qui occupent en général les postes de contrôleur financier dans les grandes communautés urbaines de Douala et de Yaoundé. Les $4 \%$ de contrôleurs financiers font partie de l'échantillon et sont formés par l'école nationale d'administration et de magistrature (ENAM); ils ne relèvent pas du MINATD. Les chefs de services de niveau $\mathrm{Bac}+5$ sont généralement des cadres techniques (ingénieurs de génie civil) formés non pas au CEFAM mais plutôt à l'école nationale des travaux publics de l'État (ENTPE). Mais on retrouve aussi des cadres de haut niveau tels que les ingénieurs architectes formés pour la plupart en France ou encore au Canada. On les retrouve surtout dans les gros services des communautés urbaines de Douala et de Yaoundé. Ils représentent environ $12 \%$ de l'échantillon. Ceux des chefs de services formés au second cycle du CEFAM $(1,7 \%$ de l'échantillon) occupent plutôt des fonctions relevant de l'administration générale dans les communes telles que l'état civil, la rédaction administrative, la réglementation des marchés publics, etc. Ils sont pour la plupart titulaires du Brevet d'études du premier cycle (BEPC) ou du certificat d'aptitude professionnelle (CAP).

Enfin, on retrouve quelques contractuels d'administration issus des autres écoles de formations telles que l'école supérieure des sciences économiques et commerciales (ESSEC) de l'université de Douala de niveau $\mathrm{Bac}+4$ et $\mathrm{Bac}+5$ ou encore de l'Institut universitaire de technologie (IUT) de niveau Bac +2 (titulaires de BTS ou encore d'un DUT). 


\section{Graphique 6 : Distribution des chefs de services selon leur niveau de formation}

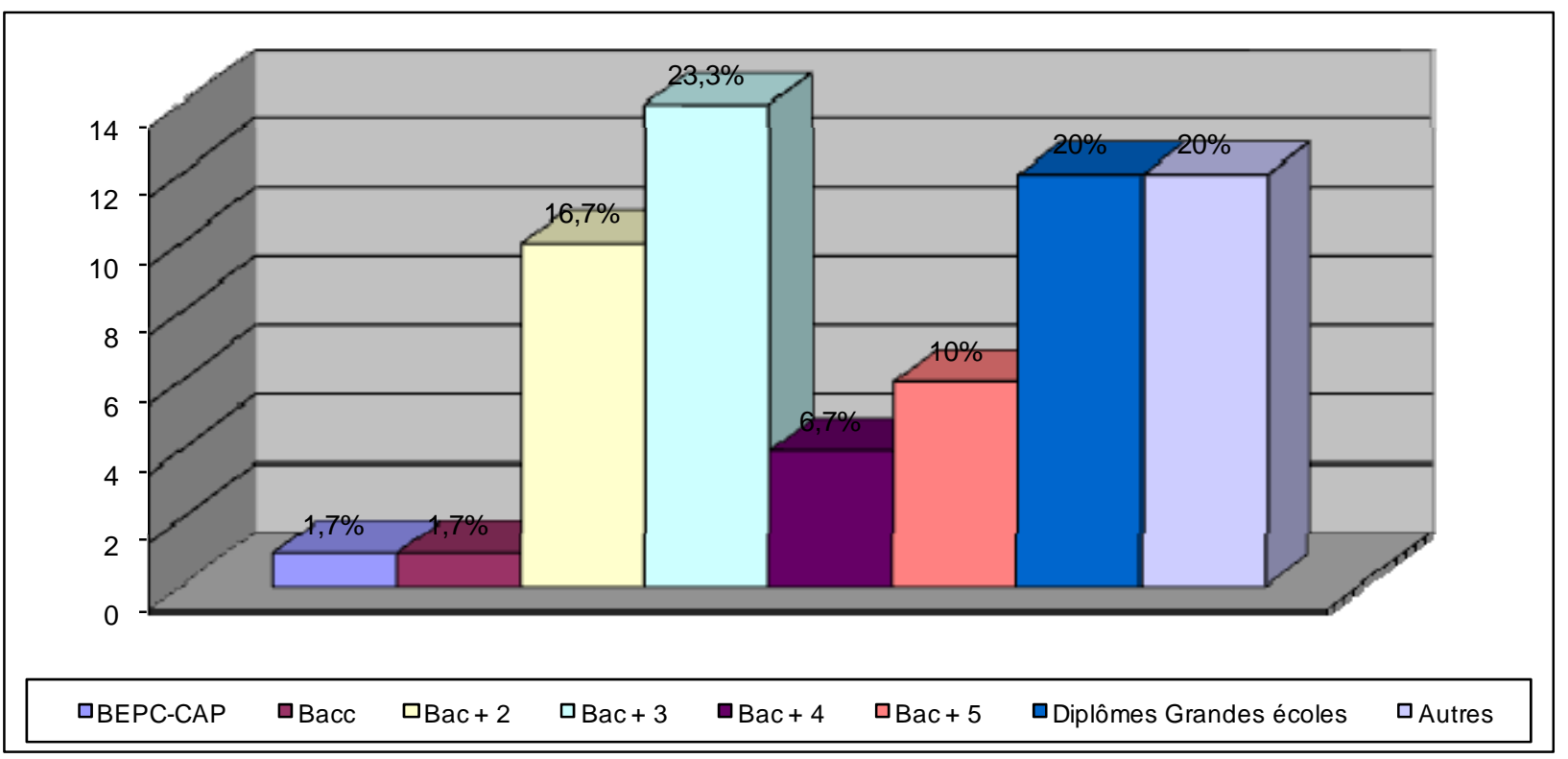

L'ancienneté assure un meilleur ajustement mutuel entre les agents qui travaillent ensemble depuis de longues années et ainsi se connaissent bien, ont pris l'habitude de s'observer, de se contrôler. Mais l'ancienneté peut aussi se révéler une entrave à l'introduction d'outils de gestion permettant d'évaluer objectivement la performance des services publics locaux ${ }^{19}$. En effet, l'évaluation vient bousculer des pratiques bien ancrées dans la vie du service. Au regard du tableau ci-dessus, 38,3\% des responsables de services ont plus de 10 ans d'ancienneté et se situe généralement dans la tranche d'âge de 40 à 50 ans ou encore de 50 à 60 ans soit respectivement $56,7 \%$ et $6,7 \%$.

\section{Graphique 7 : Distribution des chefs de services selon leur ancienneté}

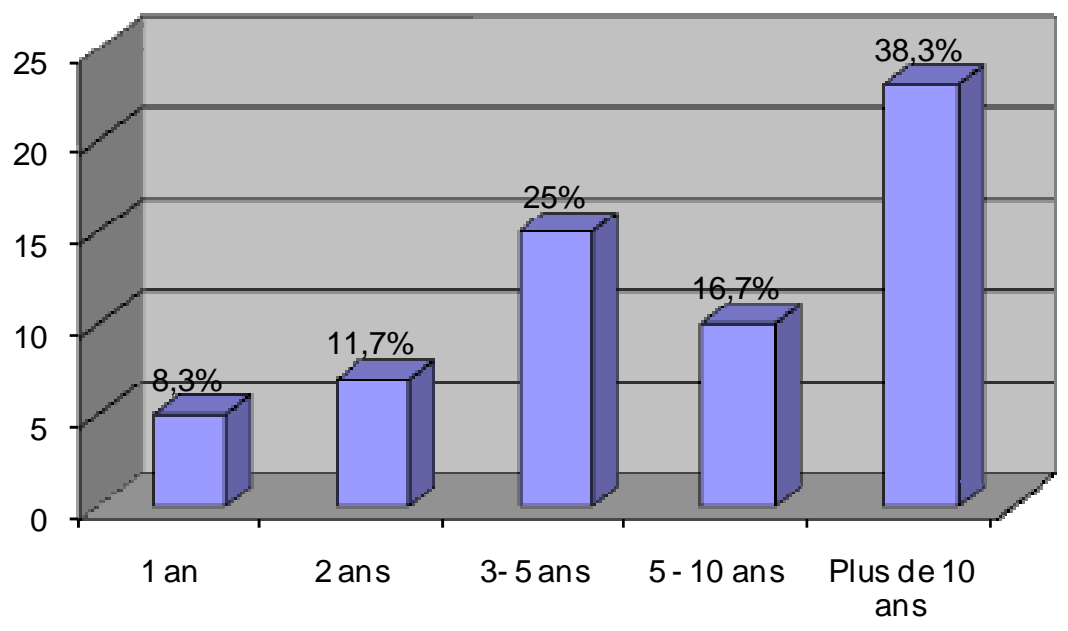

口Série1 


\section{CONCLUSION}

\section{L'analyse des données révèle que les outils de contrôle de gestion à l'instar de la comptabilité analytique n'ont pas encore pénétré le secteur municipal camerounais.}

Au terme de ce travail, il est de notre point de vue nécessaire de rappeler les grandes tendances qui se dégagent pour une meilleure compréhension des résultats que nous présentons dans notre recherche. L'étude quantitative des données recueillies nous a permis de décrire les outils de contrôle de gestion utilisés pour le suivi des objectifs. Toutefois, l'analyse des données révèle que les outils de contrôle de gestion à l'instar de la comptabilité analytique n'ont pas encore pénétré le secteur municipal camerounais. Nous avons par ailleurs observé un usage très rudimentaire des tableaux de bord au sein de ces municipalités. In fine, l'étude nous a permis de mettre en exergue un certain nombre de facteurs contingents à la fois d'ordre structurel et comportemental susceptibles d'influencer la performance des services publics municipaux.

\section{BIBLIOGRAPHIE}

1'Ouchi W.G. (1979). «A Conceptual Framework for the Design of Organizational Control Mechanisms », Management Science, pp.883-848.

${ }^{2}$ Roussarie O. (1995). «La comptabilité analytique dans les services municipaux : des méthodes de calcul à adapter à la diversité des activités de l'organisation », actes du $16^{\mathrm{e}}$ congrès de l'Association Française de Comptabilité.

${ }^{3}$ Demeester R. (2007). «L'analyse des coûts : public et privé», Politiques et Management Public, vol.25, n³, p.102-114.

${ }^{4}$ Evraert S. et Mevellec P. (1990). «Calcul des coûts : il faut dépasser les méthodes traditionnelles », Revue Française de Gestion, mars-avril-mai, pp. 12-24.

${ }^{5}$ Leduff R. et Rigall J.J. (1999). Maire, entrepreneurs, emploi: deuxièmes rencontres Ville-management, (Bayonne, Pyrénées Atlantiques, Septembre, 1998). Editions Dalloz.

${ }^{6}$ Kopel S. (2001). « Tableaux de bord de mairie: vers l'apprentissage de la responsabilité», Revue Politiques et Management Public, n³, p.159-176.
${ }^{7}$ Naro G. (1995). «Les apports de la GRH au contrôle de gestion: une revue de la littérature anglo-saxonne», Colloque de Toulouse: Comptabilité et GRH, 12 juin, AFC et AFGRH, pp.52-66.

${ }^{8}$ Gervais M. (1997). Contrôle de gestion, Economica, Paris.

${ }^{9}$ Mendoza C. (1998), «Mettre en place des tableaux de bord de gestion», La revue fiduciaire comptable, juillet/août, p.35-62.

${ }^{10}$ Demeester R. et al. (1997). Le contrôle de gestion dans le secteur public, Paris : LGDJ.

${ }^{11}$ Nizet J. (1992). « Les gestionnaires face à l'efficacité et à l'efficience », Gestion 2000, n 1, pp.73-85.

${ }^{12}$ Le Duff R. et Papillon J.C. (1988). Gestion publique, Editions d'organisations, Vuibert, Paris.

${ }^{13}$ Meyssonier F. (1991). «Contrôle de gestion communal : les conditions de l'efficacité", Revue Française de Gestion, Septembre-Octobre, pp.54-62.

${ }^{14}$ Bartoli A. (1997). Management public, Paris : Dunod.

${ }^{15}$ Langevin et al. (1995). "Contrôle de gestion et ressources humaines: une nécessaire collaboration pour mieux gérer la performance $»$, actes du $6^{\mathrm{e}}$ congrès de l'AGRH, Poitiers, pp. 189-196.

${ }^{16}$ Bouquin H. (1997). b - «Contrôle» Joffre P. et Simon Y., Encyclopédie de gestion, tome 1, Economica, Paris, article 33, pp. 667-685.

${ }^{17}$ Avelé D. (2009). «L'évaluation et le contrôle de la performance des services publics municipaux des villes camerounaises: une approche contingente », Ateliers de production d'articles, IGR-IAE/CIDEGEF.

${ }^{18}$ Nyengue E.P. (2006). L'organisation du système d'information comptable des entreprises camerounaises: Essai d'observation et interprétation des pratiques, Thèse de doctorat en sciences de gestion, Université Montesquieu-Bordeaux IV.

${ }^{19}$ Avelé D. (2009). L'évaluation et le contrôle de la performance des services publics municipaux des villes camerounaises, Thèse de doctorat en sciences de gestion, Université Montesquieu Bordeaux IV, Juin. 


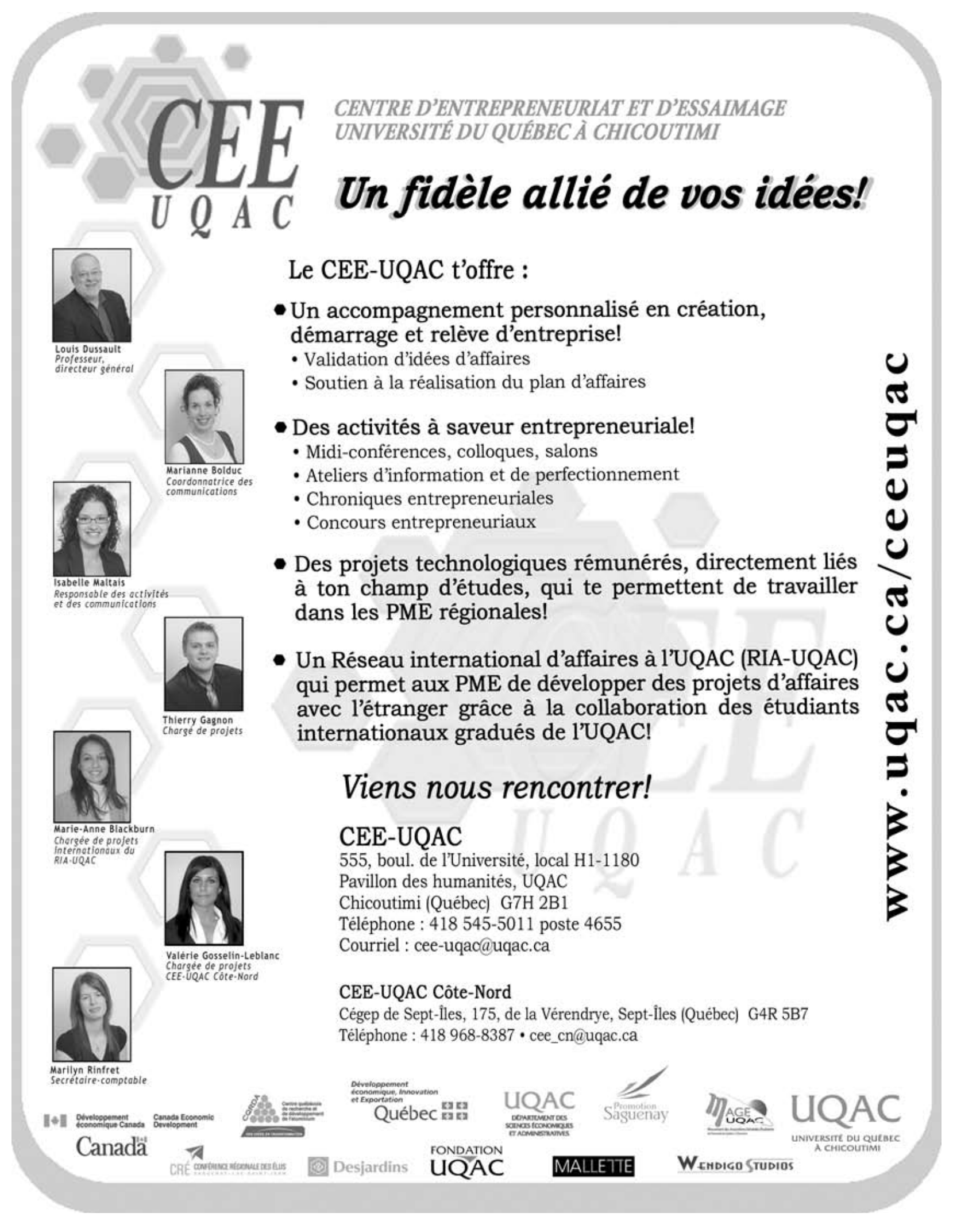

